strains were mainly isolated from treated or relapsed patients with Promine- or DDS-administration for a long time.

Even the sensitive strain (no growth was observed in $0.1 \mathrm{mg}$ diet/gram) was not completely suppressed in the case of DDS-administration which was given from the time of 3 to 6 months after the inoculation with M. leprae, these strains were mainly isolated from the untreated patients.

On the other hand, DDS-administration to the moderately resistant strain in the mouse foot-pad (slightly growth in $0.1 \mathrm{mg}$ diet/gram) was continued in a period of about 14 to 20 months, this strain was reached to the plateau level (approximately $10^{5}$ to $10^{7}$ bacilli/foot-pad).

The inhibitory effect of Kanamycin and Streptomycin against DDS-resistant strain was not completely suppressed than the DDS-sensitive strain, which was received with each dosage of $0.4 \mathrm{mg}$ per mouse three times a week from the time of 2 to 6 months after the inoculation with these strain.

Differences of the activity of anti-cancer drugs (Mitomycin C, Hydroxyurea, Toyomicim, 5-Fluorouracil) against DDS-resistant strains and DDS-sensitive strains were not observed in mouse foot-pad system.

\title{
MEDICAL REHABILITATION OF LEPROSY
}

\author{
TATSUJI TAMAI \\ Department of Orthopaedic Surgery, Kumamoto University \\ Medical School, Kumamoto, Japan
}

In Japan, a substantial number of leprosy patients has been rehabilitated to society from the leprosaria, owing to their dramatic response to promin and D. D. S., and to the improvement of orthopaedic treatment for disturbance of function and deformity of extremities.

The problem of personality in leprosy is very important not only for social recovery, but also medical rehabilitation as in the case of other crippeldness.

The observation of personality tells us that the patients differ a great deal from healthy persons in terms of somatic inferiority complex.

According to our experiences, medical rehabilitation of leprosy should be begun immediately after the disease is diagnosed. A success in the rehabilitation of leprosy can only result from cooperation of specialists for leprosy in different fields, besides basic medical treatments of the disease.

Physical therapy is a valuable treatment, using oil massage, vibra-bath, paraffin-bath, under-water exercise and electric treatment and so on. In the case of paralysis of the hands and feet, an early employment of physical therapy has been found extremity valuable and has contributed to recovery of paralysis and to prevention of deformities.

The reconstructive surgery is very effective not only to improve the activity of daily life of patients, but also to remove their inferiority complex, through the 
reconstruction of deformity and restoration of function.

It is no doubt that the reconstructive surgery is very valuable, but it is not the best for all the cases with changes in hands and feet. Use of a splint or a device for disfunction and deformity will have a favorable effect comparable to and sometimes better than reconstructive surgery. Even amputation should be undertaken to provide good function for some cases with notable changes on the foot, and the prerequisite is to assure the condition for ideal prothesis.

And the plastic surgery for the severe deformity of face is very important procedure to remove the inferiority complex.

The efficient medical rehabilitation including reconstructive surgery must be employed, along with basic medical treatment of this disease.

\title{
THE PRESENT STATUS OF LEPROSY IN KOREA IN 1969
}

\author{
JoON LEW \\ Yonsei University, Soel, Korea
}

The earliest record of leprosy in Korea goes back to around the end of 13th century. By the end of World War II, under the Japanese administration, about 8,000 leprosy patients were institutionalized under the concept of segregation. By 1968, a total of known, registered leprosy cases in Korea sums 37,571 among which 7,479 are institutionalized and 30,092 are being treated as domiciliary patients either at home or in settlement and in resettlement villages.

The modern western medicine was introduced to this country in early 20th century. In 1909 Rev. Drs. W. H. Forsythe and R. M. Wilson founded the first private leprosarium in Kwangju and this was the very beginning of introduction or modern medicine into the treatment of leprosy patients in this country.

During the past two and a half decades, many significant progresses have been made in the treatment of leprosy in Korea. The sulfone drugs were introduced in this country in 1953.

In 1955, a leprosy out-patients clinic, and in 1957, a leprosy mobile clinic were started.

In 1963, the over-all compulsory segregation law was abolished, and this resulted a great shift from the previous compulsory institutionization of the patieents, and leprosy out-patient skin clinics and University hospitals have been engaged in the treatment of domiciliary patients.

Following the initiation of leprosy mobile service by Catholic Mission in 1957, additional government or voluntary mobile teams have been established and these have been actively participating in case finding, treatment of home-patients and publicity campaign in rural areas.

In response to sudden increase in the number of beggar leprosy cases appeared on the streets from hinding, due to loose administrative control in 1946, after the end of the World War II, the Korean Leprosy Prevention Association, the forerun- 